\title{
Dunoso, dunare o dunale?
}

\author{
Valeria Della Valle
}

PUBBLICATO: 04 FEBBRAIO 2020

\section{Quesito:}

Alcuni lettori ci chiedono se per riferirsi alla duna costiera sia corretto l'aggettivo dunale o dunare oppure dunoso: si dice ambiente, ecosistema dunale, dunare o dunoso?

\section{Dunoso, dunare o dunale?}

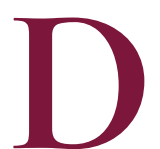

ei tre aggettivi i dizionari contemporanei, sia quelli storici sia quelli dell'uso, hanno registrato finora solo dunoso, con il significato di 'che ha i caratteri della duna, o che è costituito di dune, ricco di dune', e con l'aggiunta degli esempi deserto dunoso, litorale dunoso, coste dunose, territori dunosi ecc. L'aggettivo dunoso è datato nei dizionari, come prima attestazione, 1956, ma se ne trova già la presenza negli "Atti del primo convegno tecnico sugli Indirizzi produttivi della trasformazione agraria nel Mezzogiorno" (Istituto Poligrafico dello Stato, I954, p. I88): "Dando uno sguardo alle superfici ancora disponibili e riscattabili eventualmente alla coltura, è dato di constatare che lungo i litorali esistono zone dunose ed arenili abbandonati in quanto ritenuti sterili ed inadatti alla coltura". Passando alla stampa, in un articolo del "Corriere della Sera" del iz novembre I960, p. I6, si legge: "Per accelerare il deflusso delle acque alluvionali, ora che l'entrata è bloccata, è stata pure allargata la breccia aperta in località Bacuco nell'argine dunoso". Lunica attestazione letteraria rintracciata è tratta dal libro di Fabio Fiori, Adriatico, piccole storie di mare e di costa, (Roma, Nuova iniziativa editoriale, 2003), in cui l'autore ha scritto: "Ma sulla riva del mare, nei giorni di nebbia, le ombre evocano ricordi e può capitare di scambiare il fronte alberghiero con quello dunoso".

Più tardi accanto a dunoso si sono diffusi anche gli aggettivi dunare e dunale. Se ne trovano numerose attestazioni nella stampa. Per esempio, nel "Corriere della Sera" del 7 giugno i99i, p. 36: "In tutta la regione - si afferma in una nota - su oltre 270 chilometri di costa non ne rimangono che una ventina di ambienti dunari ancora parzialmente integri lungo il litorale del Parco Nazionale del Circeo". E il I2 ottobre 2002, sempre nel "Corriere della Sera" (Roma, p. I) Fulco Pratesi scriveva: "Sistema dunale che in pochi anni rischia di essere del tutto devastato".

Le attestazioni di dunoso, dunare e dunale nella stampa quotidiana si infittiscono nel corso degli anni, e anche nella letteratura specialistica del settore i tre aggettivi sono ben documentati (se ne trovano numerose attestazioni nell'Enciclopedia Treccani online), ma negli ultimi anni dunale e dunare sembrano prevalere numericamente sudunoso, termine forse avvertito come meno tecnico. In effetti, una differenza di significato tra dunoso e dunale/dunare ci sarebbe: infatti, gli aggettivi formati col suffisso oso "esprimono la presenza, la rilevanza, la qualità, l'effetto di ciò che è indicato dal sostantivo di base" (GRADIT), quelli in -ale e in -are indicano invece una semplice relazione con il sostantivo di base; dunque dunoso significa 'ricco di dune', caratterizzato dalla presenza di dune', mentre dunale o dunare 'proprio della duna', 'relativo alle dune'.

La diffusione di dunale può essere favorita anche dalla quasi coincidenza con l'inglese dunal, usato nella letteratura scientifica in lingua inglese.

In conclusione, si tratta di tre aggettivi tutti corretti, formati regolarmente. 


\section{Cita come:}

Valeria Della Valle, Dunoso, dunare $o$ dunale?, "Italiano digitale", 2019, XII, 2020/1 (gennaio-marzo) DOI: 10.35948/2532-9006/2020.3226

Copyright 2019 Accademia della Crusca

Pubblicato con licenza creative commons CC BY-NC-ND 